\title{
Evaluación de la implantación de las aplicaciones móviles en las universidades españolas
}

\author{
María Pinto-Molina*, David Caballero-Mariscal**, Francisco Javier García-Marco*** \\ *Departamento de Información y Documentación, Universidad de Granada. \\ Correo-e: mpinto@ugr.es | ORCID iD: https://orcid.org/0000-0002-3199-4509 \\ **Departamento de Humanidades y CCSS. Universidad de Granada. \\ Correo-e: davidcaballero@ugr.es | ORCID iD: https://orcid.org/0000-0001-6451-6047 \\ ***Departamento de Ciencias de la Documentación e Historia de la Ciencia. Universidad de Zaragoza. \\ Correo-e: jgarcia@unizar.es | ORCID iD: https://orcid.org/0000-0002-6241-4060
}

Recibido: 13-02-20; 2a versión: 23-03-20; Aceptado: 27-03-20; Publicado: 18-02-21

\begin{abstract}
Resumen: Se analiza el nivel de desarrollo e implementación de las aplicaciones móviles en las universidades españolas. A partir de una categorización y clasificación temática de las apps existentes, se diseña un instrumento de evaluación consensuado por expertos, denominado MappUn (Métrica para Mobile Apps en Universidades), que recoge las áreas funcionales, los servicios y su ponderación, para valorar la cantidad y tipo de aplicaciones móviles, y detectar las áreas de desarrollo avanzadas o rezagadas. Finalmente, se calcula el grado de visibilidad de las universidades en función del número y nivel de desarrollo de las aplicaciones móviles analizadas, permitiendo la identificación de líderes a partir de los cuales derivar experiencias significativas, mejores prácticas y consejo. Se constata que las universidades españolas se encuentran todavía en estadios iniciales de su implementación o en una fase de desarrollo moderado. La simplicidad, claridad, rigor y flexibilidad de este instrumento permite su aplicación a diferentes contextos académicos.
\end{abstract}

Palabras clave: m-Learning; comunicación móvil; teleformación; transformación digital; universidades; instrumentos de evaluación; rankings de universidades; España.

\section{Assessment of the implementation of the mobile apps in Spanish Universities}

Abstract: In this paper, the level of development and implementation of mobile applications in Spanish universities is analyzed. First, we propose a categorization and service-oriented classification of the existing apps. Second, an assessment tool was designed. This instrument, called MappUn (Metric for Mobile Apps in Universities), includes functional areas, services and their weighting, with the purpose of assessing both the quantity and type of mobile applications, and detecting the most advanced or laggard areas of development. Finally, am-Learning ranking for Spanish universities was calculated according to the number of the mobile applications provided by them for the different services, allowing for the identification of leaders from which to derive significant experiences, best practices and advice. It was concluded that Spanish universities are still in the initial stages of $\mathrm{m}$-Learning implementation or in a moderate development phase. Due to its simplicity, clarity, rigor and flexibility, this instrument can be applied to different academic contexts.

Keywords: m-Learning; mobile communication; e-Learning; universities; digital transformation; assessment tools; higher education rankings; Spain.

Cómo citar este artículo/Citation: Pinto-Molina, M.; Caballero-Mariscal, D., García-Marco, F. J. (2021). Evaluación de la implantación de las aplicaciones móviles en las universidades española. Revista Española de Documentación Científica, 44 (1), e286. https://doi.org/ 10.3989/redc.2021.1.1755

Copyright: (c) 2021 CSIC. Este es un artículo de acceso abierto bajo los términos de la licencia de uso y distribución Creative Commons Reconocimiento 4.0 Internacional (CC BY 4.0). 


\section{INTRODUCCIÓN Y ESTADO DE LA CUES- TIÓN}

\subsection{Introducción}

El aprendizaje móvil (m-Learning), enmarcado en el exponencial crecimiento en el uso de las tecnologías móviles y sus aplicaciones (tanto smartphones como sus apps), es una tendencia imparable en el ámbito universitario a nivel mundial (Krull y Duart, 2018). Debido a los cambios que provoca en las estrategias y relación con la información, influye en la motivación, calidad e incluso resultados de la educación superior (Klimova, 2019). También en España, el desarrollo de las tecnologías móviles en el contexto de la enseñanza se ha incrementado considerablemente desde 2010, principalmente entre la población más joven (Porcar y otros, 2010; Sánchez, 2012; Gijón y otros, 2013; Calzada y Martínez, 2014).

El fenómeno del despliegue del m-Learning en la Educación Superior no ha pasado desapercibido en los análisis estratégicos sobre su futuro. Así, el Informe Horizon (Adam Becker y otros, 2017) predecía para el periodo 2017-2021 un escenario de la Educación Superior caracterizado por tres tendencias-clave -enfoque en el aprendizaje colaborativo y combinado, rediseño de espacios de aprendizaje y avance de la cultura de la información- ; cinco desafíos significativos - mejora de la alfabetización digital, integración de la enseñanza formal e informal, superación de la brecha en los resultados, obsolescencia del conocimiento y un replanteamiento del papel del profesorado-profesionales de la educación-; y cuatro logros relevantes -el impulso de la tecnología para el aprendizaje móvil, el impacto del internet de las cosas, el surgimiento de la próxima generación de sistemas de aprendizaje móvil; y el progreso de la inteligencia artificial, así como el desarrollo de las interfaces intuitivas de fácil manejo, esto es, amigables (userfriendly)-. Como se puede apreciar, la adopción de las tecnologías móviles se contemplaba de forma específica en este informe de referencia en dos de los cuatro logros, es decir, de las herramientas que pueden permitir abordar los desafíos presentes en el mundo de la Educación Superior.

Sin embargo, a pesar de calificarse como solución, la adopción de las tecnologías móviles en la educación superior plantea también su propio reto: encontrar estrategias y acciones capaces de hacer más efectivas las distintas funciones de la educación superior. Como señalan Witt y Gloerfeld (2018), la implementación de las tecnologías móviles, como respuesta a las nuevas necesidades, requiere del desarrollo de nuevas estrategias que permitan la adaptación a los escenarios emergentes, mediante la elaboración de planes digitales que promuevan el proceso de transformación digital en todas las áreas funcionales y servicios universitarios (gestión, formación inicial y permanente, procesos de aprendizaje, investigación, creación y difusión del conocimiento...). Para ello, resulta fundamental desarrollar instrumentos y metodologías para conocer y monitorizar cómo las universidades están dando respuesta al reto de incorporar el $\mathrm{m}$-Learning a sus diferentes funciones.

En este sentido, el objetivo general de este estudio es monitorear el nivel de desarrollo e implementación de las aplicaciones móviles (apps) en las universidades españolas mediante un instrumento de evaluación creado ad hoc, denominado MappUn (Métrica para Mobile Apps en Universidades). MappUn incorpora una base de conocimientos, una categorización y clasificación temática de las apps existentes y un cuestionario de valoración, que permiten detectar las áreas de desarrollo más avanzadas o rezagadas, trazar un mapa de visibilidad $\mathrm{m}$-Learning de las universidades, e identificar líderes de los que obtener experiencia, mejores prácticas y consejo. Debido a su simplicidad, claridad, rigor y flexibilidad, este instrumento puede ser implementado y aplicado a diferentes contextos académicos. El análisis de las múltiples apps sirve, de igual modo, de indicador de la implementación de la m-Learning en la universidad, al permitir comprobar qué aplicaciones se dedican a la docencia, bibliotecas e investigación, y cuáles tienen otra finalidad.

\subsection{Estado de la cuestión}

La aplicación de m-Learning en la educación superior se puede calificar como un frente de investigación emergente. Una búsqueda realizada el 30 de diciembre de 2019 en Web of Science con la ecuación de búsqueda "TS=(("m-learning" OR mlearning OR "mobilelearning") AND ("highereducation" OR universit*))" en las bases de datos SCI-EXPANDED, SSCI, A\&HCI, CPCI-S, CPCI-SSH, BKCI-S, BKCI-SSH, ESCI, CCR-EXPANDED e IC arrojó 1.519 artículos interesados en el tema, caracterizado como un campo de investigación altamente interdisciplinar (figura 1). Dentro de él, destacan la investigación educativa $(68,9 \%)$-con una importante presencia de la enseñanza de las disciplinas científicas (892) - y las diferentes ramas de la informática -métodos (187), sistemas (175), aplicaciones (228), inteligencia artificial (69)-, con una aportación menor pero significativa de las ingenierías electrónicas (151) y en telecomunicaciones (68), la biblioteconomía y la documentación (58) y las intersecciones interdisciplinares de las ciencias sociales (58). 
Figura 1. Peso de las distintas áreas funcionales en el conjunto de apps de las universidades españolas



Cuando se busca la misma ecuación en el campo de título, se obtiene un conjunto más reducido, de 224 artículos directamente dedicados al $\mathrm{m}$-Learning como tema principal. Las proporciones son muy semejantes, aunque crece relativamente la contribución de la biblioteconomía y documentación, y dentro de las ciencias sociales emerge netamente el campo de la gestión. De esta forma, la distribución se podría sintetizar como sigue: investigación educativa (132), distintas ramas de la informática- incluyendo aplicaciones (45), sistemas (25), teoría y métodos (21) e inteligencia artificial (9)-, biblioteconomía y documentación (15), telecomunicaciones (11), enseñanza de disciplinas científicas (11) y gestión (8).

Las citas recibidas por este conjunto seleccionado en la fecha de la búsqueda evidencian el creciente interés en el m-Learning, confirmado para el caso español por Brazuelo y Gallego (2014) a partir de un análisis de las revistas españolas de educación entre 2009 y 2013. Estos autores destacan el incremento de publicaciones al final del periodo estudiado, entre 2012 y 2013 ; y concluyen recomendando el impulso de políticas educativas, contenidos y formación docentes específicamente dirigidos en esa dirección. Tres años después, Mateus y otros, (2017) volvieron a analizar la producción científica sobre el uso y la aplicación de móviles en las universidades españolas (2012-2016), confirmando que se trata de un tema emergente, pero indicando la escasez significativa de trabajos sobre la cuestión.

Tras analizar los resultados de las búsquedas, se confirma que la gran mayoría de los artículos publican evaluaciones de la adopción y, sobre todo, de la efectividad del aprendizaje móvil y sobre sus factores psicológicos, educativos y sociales subyacentes (Crompton y Burke, 2018). Así pues, a pesar del notable y creciente volumen de literatura académica, se constata la escasez de análisis sobre el grado de penetración de los servicios de m-Learning en la Enseñanza Superior (ES), especialmente en España.

En el panorama internacional, sin embargo, existen ya varios estudios de conjunto para Estados Unidos, Canadá, Australia, Taiwán y Hong Kong que constituyen una referencia ineludible.

En 2014, Bomhold realizó una investigación sobre los servicios móviles y las apps en las bibliotecas norteamericanas. Para ello, diseñó un cuestionario que aplicó a una muestra de bibliotecas pertenecientes a la Canergie Foundation. Comprobó que muchas bibliotecas ofrecían una gran variedad de apps, pero otras no. Al año siguiente, Bomhold (2015) profundizó en cinco de sus funciones-servicios clave: catálogos con acceso móvil (MOPAC), bases de datos de revistas, pregunta al bibliotecario (referencia), reservas para cursos de formación, y guías temáticas; y llegó a la conclusión de que "while most libraries have embraced the idea of mobile library service being central to 
their service plan, almost 30 percent still only offer a bare minimum" (pp. 36-37).

También ese año, Canuel y Crichton (2015) publicaron un análisis detallado de cómo las universidades canadienses afrontaban el rápido crecimiento y difusión de apps como elemento de apoyo en el proceso de enseñanza-aprendizaje y en la investigación. Para ello, desarrollaron una encuesta dirigida a las bibliotecas universitarias de la Association of Universities and Colleges of Canada. Buscaban conocer las apps más utilizadas, subcategorización y los servicios que ofrecían. Poco después, Canuel y sus colaboradores (2017) abordaron un estudio más concreto sobre el uso de las apps en la alfabetización digital y, a través de ella, en la mejora del rendimiento académico.

Este último año, Cheng y otros (2017) analizaron diversas universidades de Taiwán e identificaron sus respectivas apps, distinguiendo entre apps móviles y webs y sugiriendo una categorización de sus servicios. Comprobaron que la mitad de las universidades tenían sus propias apps, y que las más utilizadas eran las relacionadas con la información general y los servicios de bibliotecas. También ese año, Pechenkina (2017) llevó a cabo un análisis de las apps ofrecidas por las universidades australianas para diversos temas (herramientas de aprendizaje, organización académica, marketing o matriculación); y los resultados mostraron que la mayoría de los estudiantes ponía sus expectativas en apps no docentes.

Más recientemente, Wai y otros (2018) publicaron un estudio sobre el uso de las apps en titulaciones oficiales técnicas, economía y educación de Hong Kong. Analizaron las actitudes hacia la enseñanza móvil, la aceptación de aplicaciones para el desarrollo de la metodología cooperativa, y la incorporación e integración de estas nuevas herramientas. Concluyeron que hay una gran aceptación de las tecnologías móviles, y que las universidades deben adaptarse a este nuevo contexto, dando una respuesta efectiva.

En conclusión, aunque numerosas investigaciones han analizado el papel de las apps en la educación superior en tanto que éstas son parte integrante de los procesos de enseñanza-aprendizaje (m-Learning) (por ejemplo, entre otras recientes, Padilla y otros, 2019; Sung y otros, 2019); sin embargo, tienen generalmente un alcance muy concreto: ciertos medios (videocasts, realidad aumentada...), temas (alfabetización informacional, enseñanza de las ciencias...) o variables relevantes para explicar su uso efectivo por parte de los docentes y estudiantes (motivación, disponibilidad, capacidades técnicas e informacionales...). Por el contrario, se han encontrado menos estudios que contribuyan a levantar un mapa de la disponibilidad de herramientas m-Learning en diferentes ámbitos geográficos; y no se ha encontrado ningún estudio que aborde el desarrollo e implementación de herramientas para analizar y mapear las instituciones de educación superior en función del nivel de implantación de sus apps.

\section{MATERIAL Y MÉTODOS}

Además de confirmar la relevancia potencial de este estudio, la revisión de la literatura científica sobre m-Learning en la educación superior permitió un conocimiento profundo de experiencias relevantes en distintos contextos y países. A partir del análisis de las metodologías empleadas en dichos estudios, se identificaron las clasificaciones de apps más significativas, y se planteó una propuesta tipológica para el entorno español. Paralelamente, se elaboró una base de datos de las aplicaciones móviles localizadas en las webs de las universidades españolas. Contrastando la tipología inicial con los datos, se diseñó una versión inicial del instrumento MappUn para diagnosticar y categorizar de manera ponderada las apps de las universidades españolas, que se validó con una muestra piloto, ajustándose la versión definitiva. A continuación, el instrumento se aplicó sistemáticamente a las apps recogidas, y los datos ofrecidos se procesaron con distintos análisis descriptivos y correlacionales, mediante Microsoft Excel y SPSS Statistic 22. Finalmente, se discutieron los resultados y se derivaron las conclusiones y recomendaciones.

\subsection{Propuesta de clasificación de las apps}

En el análisis de la literatura científica sobre la cuestión se identificaron varias investigaciones que proponían clasificaciones de apps para el marco de la Enseñanza Superior (Bomhold, 2013, 2014; Vassilakaki, 2014; Canuel y Crichton, 2015; Canuel y otros, 2017; Pechenkina, 2017; y Cheng y otros, 2017) (tabla 1). Dichas clasificaciones fueron contrastadas para establecer las principales categorías (áreas funcionales) y sus aspectos (servicios). En la revisión destacaron, por orden de frecuencia, las categorías relacionadas con la información general, las bibliotecas y la gestión-procedimientos. Además, con menor prevalencia, se identificaron otras dos categorías que están en relación con el marketing y los recursos para el e-Learning, bien directamente o a través de diversas sub-categorías. Como resultado, se obtuvieron cinco categorías que constituyeron el punto de partida para el diseño inicial del instrumento: información general, bibliotecas, organización y gestión, marketing y e-Learning. 


\subsection{Recogida de datos}

La recogida de datos se llevó a cabo entre marzo y mayo de 2019, y se actualizó en septiembre de 2019. Se emplearon dos fuentes: los sitios web de las universidades, con especial atención a las bibliotecas universitarias; y la plataforma Google Play, que habitualmente incluye más apps que la App-Store de Apple. A partir de ambas fuentes, se creó una base de datos con el nombre de la universidad, su acrónimo, el título de las apps, una categorización provisional (entre dos y cuatro palabras-clave), su localización, una síntesis de su contenido y un recuento del número de apps por universidad.

Para establecer con fiabilidad el listado de universidades, se consultó el censo oficial disponible en https://www.educacion.gob.es/ruct/consultauniversidades?actual=universidades, que recoge los 87 centros de enseñanza superior oficiales reconocidos por el Ministerio. De ellos se descartaron diez que no tenían disponibles sus apps y otros once que no permitían el acceso a personal externocasi todas privadas, salvo la Universidad de Alcalá-; por lo que el análisis se llevó a cabo sobre un total de 66 universidades: 18 privadas y 48 públicas (Anexo I). El $63 \%$ de ellas (42) muestran sus apps en sus respectivos portales web.

Respecto a las apps, se localizaron un total de 237, reducidas a 212 tras descartar 25 no funcionales. Para facilitar su análisis y comprobar su funcionalidad con efectividad, se descargaron todas en un dispositivo móvil.

\subsection{Propuesta inicial del instrumento}

Para construir la propuesta inicial del instrumento, se contó un grupo de expertos. En la selección de éstos, se tuvieron en cuenta los siguientes elementos:

- Impartir docencia e investigar en el ámbito general de las Ciencias Sociales.

- Tener conocimiento y vinculación a las tecnologías móviles, a pesar de proceder de distintos campos.

- Adscripción a distintos campos de conocimiento, para garantizar la riqueza de perspectiva.

- Estar vinculados de manera permanente a la universidad.

- Mostrar una experiencia docente e investigadora reconocida.

Siguiendo las indicaciones metodológicas de Escobar-Pérez y Cuervo-Martínez (2008) y López-Meneses y otros (2018), se seleccionaron seis expertos, ya que el mínimo recomendado para garantizar la calidad del juicio es de cinco. Por otro lado, y continuando con las consideraciones de los autores, uno de ellos es especialista en técnicas de investigación (Investigación Educativa, Universidad de Granada). Del resto, dos pertenecen a las áreas de Información (Universidad de Granada y de Antioquia, Colombia), y uno respectivamente a las áreas de Educación y Tecnología Educativa de la Universidad de Granada. Los expertos fueron informados y dieron su consentimiento por escrito. Se les facilitó el instrumento y una plantilla para la valoración de los distintos componentes del instrumento.

El grupo de expertos contrastó mediante técnica de consenso (técnica Delphi de evaluación individual, puesta en común, contraste de puntuaciones y sugerencias, Reguant Álvarez y Torrado, 2016) las cinco categorías de app identificadas a partir de la literatura con una muestra de treinta apps pertenecientes a nueve universidades $(15 \%)$ seleccionadas aleatoriamente (Salamanca, Complutense, Oberta de Cataluña, Autónoma de Barcelona, Alfonso X el Sabio, Pública de Navarra, Zaragoza, Internacional de la Rioja, y Universidad Nacional de Educación a Distancia). Como resultado, las cinco categorías iniciales pasaron a diez: información general y comunicación, gestión académica y administrativa, servicios a estudiantes, bibliotecas, e-Learning, movilidad nacional e internacional, promoción y marketing, graduados y empleo, y otros.

A partir de ellas, se concretaron nueve áreas funcionales divididas en cincuenta tipos de servicios básicos, para los que computar el número de apps disponibles en cada universidad. Esas puntuaciones, tras asignarles una ponderación consensuada por los expertos, quedaron transformadas en un conjunto de indicadores o marcadores agregados a partir de los cuales obtener un ranking de áreas funcionales, servicios y/o universidades.

\subsection{Validación y pilotaje}

La versión inicial del instrumento fue validada por expertos en tecnologías móviles procedentes de las áreas indicadas. El juicio de expertos confirmó en sus rasgos generales la estructura inicial de categorías y los criterios (áreas funcionales y servicios). Los expertos, tras poner en común puntuaciones de cada categoría y exponer los aciertos y limitaciones sobre el instrumento, sugirieron tan sólo algunas recomendaciones menores sobre las áreas funcionales: redenominar la categoría Procedimientos Académicos y Administrativos utilizando un término más genérico; fundir las categorías Procedimientos Académicos y Administrativos y Graduados-Empleo; y eliminar la categoría de Otros. Como resultado, la propuesta quedó reducida a estas ocho categorías: infor- 
Tabla I. Métrica para las Apps en la Universidad (MappUn) Áreas funcionales y Servicios

\begin{tabular}{|c|c|c|c|c|}
\hline Categorías & ÁREA FUNCIONAL & $\%$ & SERVICIO & Puntos \\
\hline \multirow{4}{*}{$\begin{array}{l}\text { INFORMACIÓN } \\
\text { GENERAL }\end{array}$} & \multirow{4}{*}{$\begin{array}{l}\text { Información general y } \\
\text { comunicación }\end{array}$} & \multirow{4}{*}{10} & Directorio & 1 \\
\hline & & & Redes sociales & 4 \\
\hline & & & Medios de la Universidad -Canales & 4 \\
\hline & & & Noticias universitarias & 1 \\
\hline \multirow{5}{*}{ GESTIÓN } & \multirow{5}{*}{$\begin{array}{l}\text { GestiónAdministrativa y } \\
\text { Académica }\end{array}$} & \multirow{5}{*}{10} & Matrícula & 2 \\
\hline & & & Notas & 2 \\
\hline & & & Trámites & 3 \\
\hline & & & Espacios físicos y organización eventos & 1 \\
\hline & & & Prácticas & 2 \\
\hline \multirow{8}{*}{ SERVICIOS } & \multirow{8}{*}{ Servicios a los Estudiantes } & \multirow{8}{*}{20} & Salud & 2 \\
\hline & & & Deportes & 2 \\
\hline & & & Cultura (conferencias, charlas, talleres, etc.) & 2 \\
\hline & & & Residencias y comedores & 3 \\
\hline & & & Informática - Utilidades & 4 \\
\hline & & & Serviciosorientación - Tutorías - Psicología & 2 \\
\hline & & & Discapacidad e inclusión & 2 \\
\hline & & & Ayudas y becas & 3 \\
\hline \multirow{4}{*}{ BIBLIOTECA } & \multirow{4}{*}{ Bibliotecas } & \multirow{4}{*}{12} & Servicios de biblioteca & 4 \\
\hline & & & Gestión del préstamo y otros servicios & 2 \\
\hline & & & Catálogos y bases de datos & 3 \\
\hline & & & Formación & 3 \\
\hline \multirow{3}{*}{ e-LEARNING } & \multirow{3}{*}{ e-Learning } & \multirow{3}{*}{13} & Plataforma docente & 5 \\
\hline & & & Mobile Learning & 4 \\
\hline & & & Enseñanzas virtuales, CEVUG & 4 \\
\hline \multirow{2}{*}{ INVESTIGACIÓN } & \multirow{2}{*}{ Investigación } & \multirow{2}{*}{10} & Fondos & 5 \\
\hline & & & Proyectos y grupos & 5 \\
\hline \multirow{3}{*}{ MOVILIDAD } & \multirow{3}{*}{$\begin{array}{l}\text { Movilidad Nacional e } \\
\text { Internacional }\end{array}$} & \multirow{3}{*}{10} & Nacional & 3 \\
\hline & & & Internacional & 3 \\
\hline & & & Becas & 4 \\
\hline \multirow{5}{*}{ PROMOCIÓN } & \multirow{5}{*}{$\begin{array}{l}\text { Marketing, Promoción y } \\
\text { Egresados }\end{array}$} & \multirow{5}{*}{15} & Posgraduados e instrucción formal & 3 \\
\hline & & & Facilidades a graduados & 3 \\
\hline & & & Empleo & 3 \\
\hline & & & Innovación y emprendimiento & 3 \\
\hline & & & Aprendizaje permanente & 3 \\
\hline
\end{tabular}

mación general, gestiones, servicios, biblioteca, e-Learning, investigación, movilidad y graduados.

Igualmente, los expertos recomendaron eliminar varias sub-categorías (servicios) por resultar reiterativas o poder ser incluidas en otras: mapa del campus, agenda-eventos académicos, gestión del tiempo, técnicas de estudio, evaluación y cursos docentes, seguridad, ocio, transporte, patrimonio y turismo, anuncios clasificados, voluntariado-cooperación, impacto y visibilidad, experiencias, facilidades y currículum vitae.

Asimismo, de manera simultánea, se realizó un pilotaje sobre la muestra indicada en el apartado anterior, que confirmó también la necesidad de re- 
agrupar y renombrar algunas áreas funcionales, y de suprimir algunos servicios, debido a su baja representatividad. Como resultado, los 50 servicios iniciales quedaron reducidos a 34 (Tabla 1).

Para neutralizar la posible subjetividad de los autores, se pidió también a los expertos que valoraran las ponderaciones iniciales, observándose un alto nivel de acuerdo y consistencia interna. Las ponderaciones definitivas se determinaron a partir de los valores medios, tomando en consideración tanto las puntuaciones iniciales como la de los expertos. Como resultado, tanto las categorías como los servicios obtuvieron unas puntuaciones consensuadas y proporcionales. La puntuación de la categoría general se estableció entre 10 y 15 puntos, dependiendo del número de servicios que constituyen cada bloque, y la relevancia de los servicios en la vida académica del estudiantado o los procesos de enseñanza-aprendizaje móvil. De esta forma, los expertos consensuaron conceder un único punto de valoración a servicios comunes y básicos, como directorio, noticias y eventos; mientras que, a determinados servicios como fondos, proyectos de investigación o ayudas públicas se les otorgaron altas puntuaciones (entre cuatro y cinco), por la relevancia que tienen para el estudiantado universitario y las posibilidades que ofrecen para la comunidad universitaria. Se pretendió garantizar que la proporción de los puntos de las distintas categorías y servicios fuera equilibrada. De igual modo, se trató de que la máxima puntuación total sobre las que podrían evaluarse las universidades, en función de sus apps, fuera de 100.

\subsection{Diseño final de MappUn}

Como ya hemos señalado, MappUn es un instrumento cuyos objetivos específicos son medir el grado de introducción y desarrollo de las aplicaciones móviles en las universidades con tres objetivos: proporcionar un indicador para conocer la presencia y desarrollo de las aplicaciones móviles; ofrecer una fuente de información de las áreas funcionales y servicios universitarios recogidos por las apps; y servir como elemento de promoción y visibilidad de las instituciones.

Aunque el objeto fundamental de la presente investigación se basa en el análisis de las apps universitarias, este estudio ha de concebirse dentro del contexto de la enseñanza móvil. Si bien la m-University o universidad móvil está adquiriendo un gran protagonismo en la enseñanza superior, nuestro análisis se halla encaminado a analizar la presencia de las apps en la universidad como facilitadora de los procesos de enseñanza-aprendizaje. Al mismo tiempo, pretende describir la adecuación de la universidad española a los contextos de desarrollo de las tecnologías móviles. En este sentido, las apps de uso académico se tornan en un relevante indicador.

Desde un punto de vista práctico, se organiza en tres partes: los nombres, acrónimos, urls de las universidades, y el número y denominación de las apps; los datos relativos a las categorías, áreas funcionales, servicios y ponderaciones (Tabla I); y una sección de observaciones. A continuación, se describen brevemente las características y componentes de estas ocho áreas funcionales y sus correspondientes servicios:

1. Información general y comunicación. Se centra en los aspectos más generales, visibles y elementales de la universidad, como medio de orientación a priori (Cheng y otros, 2017), esto es, servicios como directorio, mapas, calendario de eventos, su presencia en las redes sociales, noticias y diferentes canales de comunicación.

2. Gestión académica y administrativa. Es una de las áreas más demandas por el estudiantado. Sus apps se ocupan de la burocracia, matriculación y otros trámites que requieren los diversos miembros de la comunidad académica (Pechenkina, 2017; Cheng y otros, 2017). Además, hay un espacio para las prácticas generales y específicas. En concreto incluye: inscripción-matriculación, notas, procedimientos-trámites (secretaría virtual), espacios físicos y organización de eventos (aulas ordinarias o específicas, salas para trabajar en equipo, laboratorios o aulas de prácticas) y prácticas (de grado, módulo o asignatura, prácticum).

3. Servicios a estudiantes. Cubre una gama variada de aspectos muy solicitados por toda la comunidad académica (Pechenkina, 2017; Bomhold, 2014): ocio, actividades complementarias (deportes, cultura), comedores universitarios y residencias. Además, y debido a la diversidad del estudiantado y los desafíos de la sociedad actual, incluye servicios específicos, como orientación, apoyo psicológico, inclusión y becas. Por ello, el instrumento aborda los siguientes servicios: salud, deportes, cultura (conferencias, charlas, seminarios), alojamiento (habitaciones, residencias, comedores), informática-utilidades, orientación (tutorización y servicios de psicología), discapacidad e inclusión, y becas.

4. Bibliotecas. Proporcionan un servicio, guía y orientación profesional fundamental en el proceso de formación académico-universitario de grado y posgrado (Vassilaki, 2014; 2015; Canuel y otros, 2017) y en la investigación y extensión. El instrumento detalla como servicios: préstamos, catálogos, bases de datos, así como subscripciones y renovaciones de préstamos bibliote- 
carios y programas de formación en bibliotecas (Bomhold, 2013; 2014).

5. E-Learning. Se basa en el uso generalizado de plataformas educativas y todos los elementos relacionados con la enseñanza móvil y, en términos generales, la enseñanza online/virtual (Canuel y otros, 2017; Bomhold, 2014). MappUn incorpora estos tres servicios: plataformas educativas, aprendizaje móvil y enseñanza virtual.

6. Investigación. Hace referencia a fondos, impacto, innovación y transferencia, así como materiales y medios disponibles (Canuel y otros, 2017). Además, se incluyen transferencia, resultados y proyección de futuro.

7. Movilidad. Los programas de movilidad e intercambio despiertan el interés de muchos estudiantes tanto a nivel nacional como internacional, y requieren información sobre el sistema de becas, los acuerdos entre universidades, los coordinadores y responsables, los requisitos de participación y la burocracia a seguir (Pechnekina, 2017). Los servicios de movilidad propuestos en MappUn son, para movilidad nacional, destinos nacionales, estancias breves o intercambios; y para movilidad internacional, Erasmus, Free Movers y otros convenios, y Becas.

8. Marketing, Promoción y graduados. Esta área funcional incluye distintas opciones, como posgrados, másteres y doctorados. También se refiere a la formación permanente y el empleo (Canuel y otros, 2017; Pechnekina, 2017).

\section{ANÁLISIS Y RESULTADOS}

La evaluación de las apps seleccionadas ofrece resultados en tres niveles: en primer lugar, propor- ciona un enfoque descriptivo general sobre las universidades y sus apps, considerando su tipología, frecuencia y uso; en segundo lugar, permite describir el grado de desarrollo de las áreas funcionales y servicios sin entrar en la calidad de las apps, sino valorando simplemente su disponibilidad real; y, finalmente, ofrece una clasificación de las universidades en razón del grado de implementación de sus aplicaciones móviles, distinguiendo tres grupos en función de su nivel de madurez: inicial (0-30 puntos), en desarrollo (31-70) y desarrollada (71-100).

\subsection{Resultados generales}

En lo referente al número de apps por universidad, el $37,88 \%$ de los centros universitarios tienen una sola aplicación (25 universidades), sean o no funcional o multifuncional (con diversos servicios); un $22,72 \%$ cuenta con dos ( 15 universidades); y el $39,40 \%$ ofrece al menos tres (26).

Respecto a la finalidad de las apps, 63 apps $(29,72 \%)$ son básicas o genéricas, mientras que la mayoría de las aplicaciones analizadas, 127 $(59,91 \%)$, pueden considerarse como específicas, principalmente debido a razones contextuales. Las apps pertenecientes a este último grupo tiene finalidades muy diversas. Entre las más frecuentes, se pueden subrayar dos usos concretos: las prácticas específicas y los hábitos de salud. Sólo 22 de las 212 estudiadas $(10,38 \%)$ se dedican específicamente a la enseñanza, a pesar de la creciente inserción de las tecnologías móviles en los procesos de enseñanza-aprendizaje.

Se han detectado algunas apps relacionadas con prácticas, frecuentemente para asignaturas específicas o áreas comunes de estudio. Se observa una gran heterogeneidad de apps, debido a la diversi-

Tabla II. Resumen de puntuaciones de las App por áreas funcionales

\begin{tabular}{|c|c|c|c|c|c|c|c|c|}
\hline \multicolumn{9}{|c|}{ Estadísticos descriptivos } \\
\hline & \multirow{2}{*}{ Área funcional } & \multicolumn{5}{|c|}{ Puntuaciones } & \multirow{2}{*}{$\begin{array}{l}\text { Desviación } \\
\text { estándar }\end{array}$} & \multirow{2}{*}{$\begin{array}{c}\text { No de } \\
\text { apps }\end{array}$} \\
\hline & & Mínima & Media & Máxima & Ideal & Total & & \\
\hline 1 & Información & 1 & 7.88 & 10 & 10 & 449 & 2.472 & 57 \\
\hline 2 & Gestión & 1 & 3.64 & 8 & 10 & 193 & 1.798 & 53 \\
\hline 3 & Servicios & 2 & 3.64 & 9 & 20 & 142 & 1.953 & 39 \\
\hline 4 & Biblioteca & 2 & 5.92 & 12 & 12 & 71 & 3.450 & 12 \\
\hline 5 & e-Liaoning & 2 & 5.77 & 10 & 13 & 202 & 1.942 & 35 \\
\hline 6 & Investigación & 5 & 6.67 & 10 & 10 & 40 & 2.582 & 6 \\
\hline 7 & Movilidad & 3 & 6.64 & 10 & 10 & 166 & 1.319 & 25 \\
\hline 8 & Promoción & 3 & 5.54 & 12 & 15 & 288 & 2.477 & 52 \\
\hline
\end{tabular}


dad de titulaciones. Sólo algunos grados concretos tienen apps de prácticum. Además, algunas apps genéricas incluyen elementos para las prácticas de grado, a pesar de no haber sido específicamente concebidas para este fin.

\subsection{Resultados por áreas funcionales y ser- vicios}

Los valores obtenidos con MappUn permiten valorar el tipo de aplicaciones móviles de las que disponen las universidades españolas, así como su temática concreta y el peso que tienen las distintas áreas funcionales y servicios (Tabla II). La información más detallada por Universidad figura en el Anexo II

1. Información General. Es el área funcional más frecuente (tablaII). El 86,36\% de las instituciones analizadas posee su correspondiente app en esta área. El $63 \%$ de sus descriptores puntúa por encima de ocho.

2. Gestión Académica y Administrativa. Se trata, de igual modo también de una de las áreas funcionales más significativas, presente en las apps de 53 universidades $(80,30 \%)$, cinco de ellas privadas. Ninguna universidad alcanzó la máxima puntuación prevista para este criterio, y el máximo obtenido se situó en 8 puntos (UGR y UVA).

3. Servicios a estudiantado. A pesar su relevancia, sólo el 59,09\% de las universidades (39) ofertan apps, la mayoría públicas (76,92\%). Entre ellas, de nuevo, lidera la Universidad de Granada.

4. Servicios de Bibliotecas. Solamente un $18,18 \%$ de las universidades (12) incluye apps, un resultado discreto frente a las expectativas ofrecidas en otros estudios (Cheng y otros, 2017). El ranking para este criterio está encabezado por dos universidades privadas, que obtuvieron la máxima puntuación: IE y UCAV.

5. e-Learning. El 53,03 \% de las ofrece apps para e-Learning. Sólo se hallaron ocho universidades privadas entre éstas. Aunque algunas instituciones privadas han optado por esta metodología, no pudieron ser analizadas porque sus apps no están accesibles para personal externo.

6. Investigación. Esta área obtuvo una baja puntuación, a pesar de constituir uno de los pilares de la universidad. Sólo seis universidades $(9,09 \%)$ mostraron aplicaciones relativas a la investigación; y en sólo dos casos, Politécnica de Madrid y Politécnica de Cataluña, el descriptor de este indicador alcanzó la máxima puntuación posible (10 puntos).
7. Movilidad nacional e internacional. Es, tradicionalmente, uno de los servicios más demandados por el estudiantado de las universidades españolas, según confirman las estadísticas y la publicidad de sus webs. No obstante, únicamente 25 universidades $(37,88 \%)$ aparecen en el ranking de este criterio, y dos de ellas puntuaron por debajo de 5. La atención a la movilidad interna parece baja, mientras que la movilidad Erasmus se está incrementando significativamente.

8. Marketing, Promoción y Graduados. El 78,79\% de las universidades (un total de 52) oferta apps para la promoción, egresados y marketing. Por número de universidades, esta área funcional ocupa la tercera posición. Este dato parece correlacionar con la tendencia decreciente en el número de estudiantes de nuevo ingreso. Las universidades privadas no obtuvieron mejores puntuaciones en este criterio, aunque todas cubrían esta área funcional.

En la tabla II y figura 1 se muestran los valores medios de cada una de las áreas funcionales, así como la máxima puntuación posible. En resumen, el área de mayor peso es información general; y las de menor peso, las de gestión y servicios.

En lo referente al carácter de las universidades, las privadas sólo sobrepasan a las públicas en los criterios relacionados con bibliotecas y movilidad, dos áreas muy demandadas por el estudiantado; mientras que adolecen de una total ausencia de apps de apoyo a la investigación. En el resto de las áreas, las instituciones públicas y privadas presentan valores similares.

Un amplio número de universidades incluyen apps relativas a los campos de Información General, Gestión y Marketing (más del $75 \%$ de las instituciones). No obstante, sólo en el área de información general se alcanzan las máximas puntuaciones. La función Servicios, a pesar de tener el peso relativo más alto, logró una ponderación media inferior (figura 2).

El análisis de la distribución de las puntuaciones máximas, mínimas y medias en cada área funcional ha permitido llevar a cabo inferencias a partir de dispersión de los marcadores de las distintas apps (figura 3). Las áreas funcionales que muestran mayor dispersión son aquéllas en las que las universidades presentan un grado de desarrollo muy diferente. Entre ellas, cuando la media está más cerca del mínimo - bibliotecas (4) y marketing (8)-se evidencia que una parte importante de las universidades obtuvieron puntuaciones bajas en este criterio; viceversa, cuando la media está más cerca del máximo - como información general 
Figura 2: Puntuaciones medias y número de universidades implicadas, por área funcional



Figura 3: Ranking de las universidades españolas más destacadas (global y por áreas funcionales)

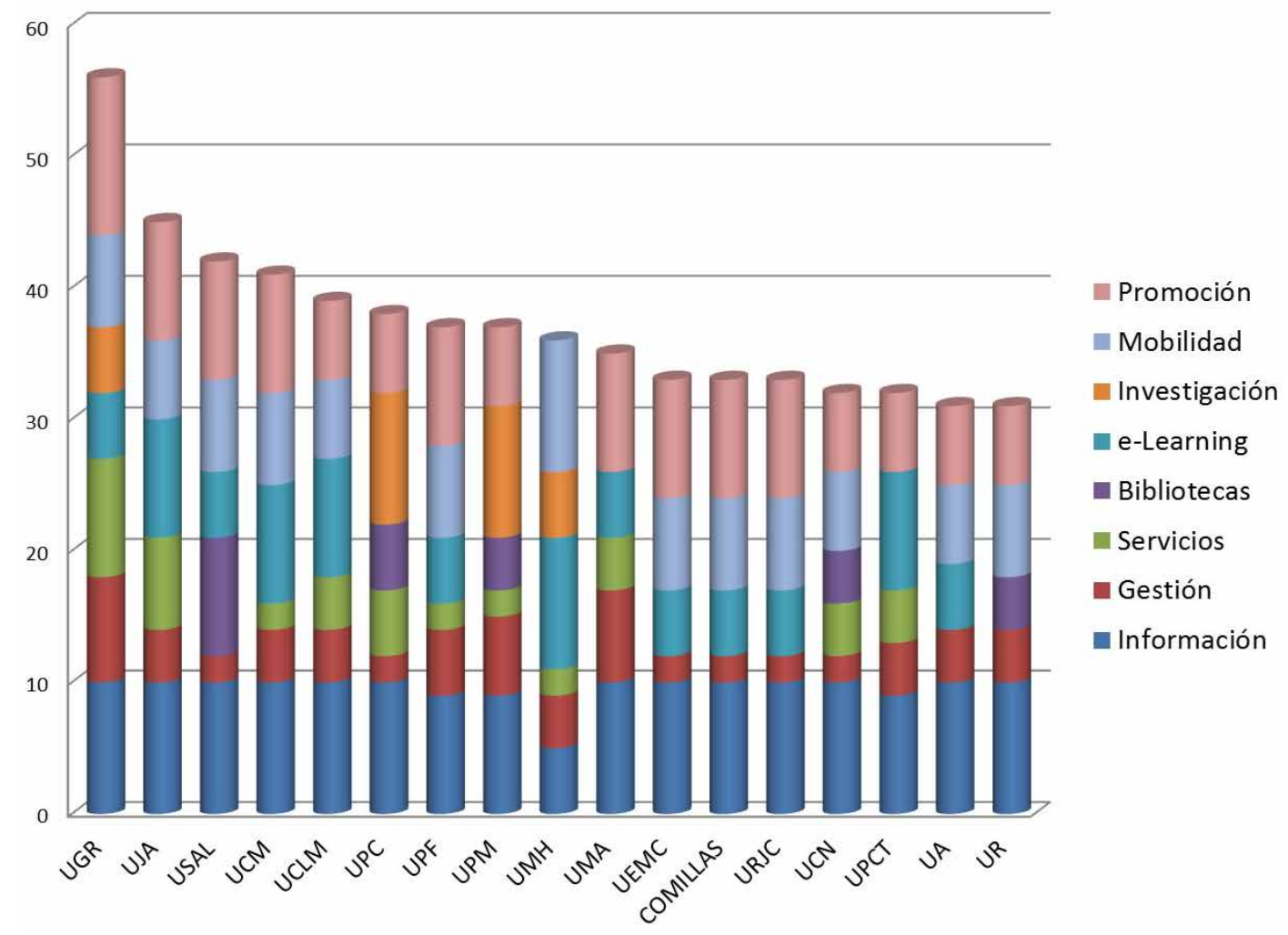


(1) - la mayoría están bien puntuadas. En el lado contrario, destaca la consistencia y uniformidad de las puntuaciones obtenidas en el área de investigación (6), la de menor dispersión global.

\subsubsection{Visibilidad de las universidades según las funciones de las apps}

De acuerdo con los resultados obtenidos, las universidades quedan clasificadas en tres grupos según su grado de desarrollo en m-Learning: la mayor parte de las universidades se incluirían en la categoría de inicial (49); 17 en desarrollo; y no se identifica ninguna perteneciente a la categoría desarrollada. En conclusión, se puede afirmar que la implementación de las apps móviles en la educación superior española se halla aún en un incipiente estado de desarrollo.

La figura 3 muestra la distribución de las diecisiete universidades en desarrollo, discriminando el peso relativo de las diferentes áreas funcionales. Ninguna universidad completa las ocho áreas funcionales propuestas por el instrumento; sólo una universidad muestra apps en siete de las ocho áreas; siete universidades reúnen seis criterios; y el resto se halla por debajo de estas puntuaciones.

\subsubsection{Correlaciones entre las áreas funcionales}

El análisis de correlaciones bivariadas permite demostrar la independencia entre la mayor parte de las ocho áreas funcionales, que puede comprobarse en la falta de significatividad de la mayoría de las correlaciones (Tabla III). Sin embargo, se produce una correlación fuerte y altamente significativa entre las áreas de biblioteca y e-Learning, y menor pero significativa entre las áreas de promoción, información y movilidad. Se dan también correlaciones significativas, aunque moderadas, entre el número de apps y las puntuaciones medias de las áreas funcionales de gestión, promoción y e-Learning, así como con el marcador total de cada universidad. También entre la puntuación total y las áreas de información, e-Learning, y promoción.

La futura aplicación del instrumento MappUn en otros conjuntos de datos podría permitir comprobar si estas correlaciones son estadísticamente significativas entre ambas áreas funcionales: bibliotecas y e-Learning, por un lado; y movilidad y promoción, por otro; o si por el contrario, sólo responden a las características particulares del conjunto de datos que hemos analizado. Si no fuera así, sería necesario proceder a una revisión de la estructura del instrumento por medio de la integración de cada uno de estos pares de áreas, fusionados en una sola.

\section{DISCUSIÓN Y CONCLUSIONES}

Esta investigación pionera ha tratado de cubrir el vacío que existe en publicaciones científicas que aborden el uso y distribución espacial y temática de las aplicaciones móviles en Educación superior, especialmente en el caso de las universidades españolas, que contrasta con el incremento de la presencia, utilidad e impacto en la

Tabla III. Correlaciones significativas entre áreas funcionales, número de apps, y puntuación total de cada universidad

\begin{tabular}{|c|c|c|c|c|c|c|c|c|c|c|c|}
\hline \multicolumn{12}{|c|}{ Correlaciones de Pearson } \\
\hline & & $\stackrel{1}{1}$ Información & $\stackrel{2}{\text { Gestión }}$ & $\stackrel{3}{3}$ Servicios & $\begin{array}{c}4 \\
\text { Biblioteca }\end{array}$ & $\begin{array}{c}5 \\
\text { e-Learning }\end{array}$ & $\begin{array}{c}6 \\
\text { Investigación }\end{array}$ & $\begin{array}{c}7 \\
\text { Movilidad }\end{array}$ & $\begin{array}{c}8 \\
\text { Promoción }\end{array}$ & $\begin{array}{l}\text { No de } \\
\text { Apps }\end{array}$ & $\begin{array}{l}\text { Puntuación } \\
\text { total }\end{array}$ \\
\hline 1 & Información & 1 & -.140 & .306 & .442 & -.299 & .631 & -.179 & ,376* & .002 &, $661^{* *}$ \\
\hline 2 & Gestión & & 1 & .028 & -.569 & -.058 & -.408 & .091 & .239 &, $357 * *$ & .140 \\
\hline 3 & Servicios & & & 1 & .116 & -.050 & -.257 & -.183 & .260 & .152 & .311 \\
\hline 4 & Biblioteca & & & & 1 & $1,000 * *$ & .c & .500 & .491 & -.408 & -.198 \\
\hline 5 & e-Learning & & & & & 1 & .c & .336 & .180 & ,370* & ,339* \\
\hline 6 & Investigación & & & & & & 1 & .c & 0.000 & -.286 & .173 \\
\hline 7 & Movilidad & & & & & & & 1 & ,623** & .359 & .167 \\
\hline 8 & Promoción & & & & & & & & 1 &, $356 * *$ &, $744 * *$ \\
\hline & No de Apps & & & & & & & & & 1 &, $443 * *$ \\
\hline & $\begin{array}{l}\text { Puntuacion } \\
\text { total }\end{array}$ & & & & & & & & & & 1 \\
\hline & \multicolumn{9}{|c|}{ *. La correlación es significativa en el nivel 0,05 ( 2 colas). } & & \\
\hline & \multicolumn{9}{|c|}{ **. La correlación es significativa en el nivel 0,01 ( 2 colas). } & & \\
\hline
\end{tabular}


vida académica de las apps universitarias (Brazuelo y Gallego, 2014; Mateus y otros, 2017). Este hecho resulta relevante para analizar y describir la enseñanza móvil superior, ya que más allá de las prestaciones que pueden ofrecer las distintas apps, el objeto de éstas debe ir encaminado al desarrollo y la actualización de la enseñanza móvil (m-Learning).

Sus aportaciones principales han sido el desarrollo de un instrumento de evaluación y ordenación de los centros de educación superior en su desarrollo m-Learning; y un mapa de la situación en España. El instrumento MappUn se ha mostrado eficaz para contribuir a conocer el nivel de implicación de las universidades españolas en las tecnologías móviles, y, por ende, en el desarrollo de apps y la implementación de la m-Learning.

Por lo que se refiere al estado del aprendizaje móvil en la educación superior española, se constata que las universidades españolas se encuentran todavía en estadios iniciales de su implementación o, en el mejor de los casos, en una fase de desarrollo moderado. Ninguna universidad dio respuesta a la totalidad de las áreas funcionales, si bien, una de ellas, lo hizo en siete.

Encabezan el ranking las universidades de Granada y Jaén. La primera se halla entre las universidades más grandes y antiguas de España, en contraste con la segunda, que, pequeña y relativamente joven, constituye un ejemplo de apuesta por el m-Learning para alcanzar mayor visibilidad y promoción. A priori, podría esperarse que la mayor parte de las universidades, situadas en mejores posiciones en los rankings de calidad internacional, ofrecieran un mayor número de apps. No obstante, algunas de las mayores instituciones educativas superiores sólo ofrecen unas cuantas apps de carácter genérico.

En general, se ha observado también que las universidades con un número significativo de grados tienden a tener un mayor número de apps. Así pues, el número de alumnos matriculados y la oferta de grados universitarios parecen ejercer una influencia significativa en la implementación o disponibilidad de apps. Además, las universidades que ofrecen enseñanza en línea o combinada tienden a tener tres o más aplicaciones.

Respecto a las áreas funcionales, el área más cubierta ha sido la de información general, coincidiendo con Cheng y otros (2017); mientras que las que ocupan las posiciones más discretas son las de gestión (en contraste con Pechenkina, 2017) y servicios. Las mayores oportunidades de mejora se dan precisamente en estas dos áreas, así como en las de promoción (a diferencia de Pechenkina, 2017) y bibliotecas, en este último caso por comparación con otros estudios internacionales (Bomhold, 2014 y 2015; Canuel y otros, 2015 y 2017; Cheng y otros, 2017; Pechenkina, 2017).

Por lo que se refiere a las apps, la mayoría tienen un propósito específico; aunque las apps genéricas (empleadas para trámites generales) son también importantes. En algunos casos, se usan exclusivamente para la universidad que las ha implementado.

En lo referente a las limitaciones de este estudio, se debe resaltar que varias universidades, en su mayoría privadas, quedaron fuera del análisis por imposibilidad de acceder a sus respectivas apps, pues no dan acceso libre a sus aplicaciones debido a razones de privacidad o exclusividad. Su inclusión permitiría un análisis más completo y una comparativa fiable entre universidades públicas y privadas.

Además, dada la escasez de apps en el área de biblioteca, que es precisamente la que mayor desviación estándar ofrece, así como su correlación altamente significativa con el área de e-Learning, probablemente fuera acertada la fusión de ambas en futuras versiones del instrumento. Los resultados de la herramienta MappUn no mostraron homogeneidad en las puntuaciones, aunque estos registros y el número de apps por universidad están moderadamente correlacionadas.

Finalmente, desde una posible prospectiva de investigación se propone profundizar en la herramienta MappUn, incorporando algunos elementos de contenido y formales, que permitan evaluar la calidad de las apps en relación a sus contenidos y uso.

No obstante, las limitaciones reseñadas, el alto porcentaje de centros de enseñanza superior y aplicaciones móviles analizados ofrecen una perspectiva muy descriptiva de la situación española en aplicaciones móviles para la educación superior, que es susceptible de contribuir a la mejora en la calidad de su implementación, y que puede ser aplicable a otros contextos universitarios.

\section{AGRADECIMIENTOS}

Este artículo publica resultados de investigación del proyecto CSO2016-80147-R (MINECO/FEDER) y ha contado con la colaboración del proyecto CSO2015-65448-R (MINECO/FEDER).

\section{ACKNOWLEDGEMENTS}

This article publishes research results from the CSO2016-80147-R (MINECO / FEDER) project and has had the collaboration of the CSO2015-65448-R (MINECO / FEDER) project. 


\section{REFERENCIAS}

Adam Becker, S.; Cummins, M.; Davis, A.; Freeman, A.; Hall, C. G.; Ananthanarayanan, V. (2017). NMC Horizon Report: 2017 Higher Education Edition, pp. 1-60. The New Media Consortium.

Bomhold, C. (2013). Educational use of smart phone technology: A survey of mobile phone application use by undergraduate university students. Program, 47(4), 424-436. https://doi.org/10.1108/PROG-01-2013-0003 [Fecha de consulta: 09/02/2020].

Bomhold, C. (2014). Mobile services at academic libraries: meeting the users' needs? Library Hi Tech,32(2), 336-345. https://doi.org/10.1108/LHT-10-2013-0138 [Fecha de consulta: 09/02/2020].

Bomhold, C. (2015). Research and discovery functions in mobile academic libraries: Are university libraries serving mobile researchers? Library Hi Tech, 33(1), 32-40. https://doi.org/10.1108/LHT-09-2014-0084 [Fecha de consulta: 09/02/2020].

Brazuelo Grund, F.; Gallego Gil, D. J. (2014). Estado del mobile learning en España. Educar em revista, (4). https://doi.org/10.1590/0104-4060.38646 [Fecha de consulta: 09/02/2020].

Calzada, J.; Martínez Santos, F. (2014). Competencia en el Mercado de banda ancha móvil en España. CuadernosEconómicos del ICE, 88, 145-177. [Fecha de consulta: 09/02/2020] https://doi.org/10.32796/ cice. 2014.88.6088.

Canuel, R.; Crichton, C. (2015). Leveraging apps for research and learning: a survey of Canadian academic libraries. Library $\mathrm{Hi}$ Tech, 33(1), 2-14. https://doi. org/10.1108/LHT-12-2014-0115 [Fecha de consulta: 09/02/2020].

Canuel, R.; MacKenzie, E.; Senior, A.; Torabi, N. (2017). Apps for academic success. Developing digital literacy and awareness to increase usage. Education for Information, 33, 135-152. https://doi.org/10.3233/EFI160085 [Fecha de consulta: 09/02/2020].

Cheng, H. C.; Kung, T. P.; Li, C. M.; Sun, Y. J. (2017, February). The current state of mobile apps development of higher education in Taiwan. En: Advanced Communication Technology (ICACT), 2017 19th International Conference (pp. 780-786). IEEE. https://doi. org/10.23919/ICACT.2017.7890227

Crompton, H.; Burke, D. (2018). The use of mobile learning in higher education: A systematic review. Computers and Education, 123, 53-64. https://doi. org/10.1016/j.compedu.2018.04.007 [Fecha de consulta: 09/02/2020].

Escobar-Pérez, J.; Cuervo-Martínez, A. (2008). Validez de contenido y juicio de expertos: una aproximación a su utilización. Avances en Medición, 6, 27-36. https:// www.researchgate.net/profile/Jazmine_Escobar-Perez/publication/302438451 Validez de contenido_y_juicio_de_expertos_Una_aproximacion_a_su_ utilizacion/links/59a8daecaca27202ed5f593a/ Validez-de-contenido-y-juicio-de-expertos-Una-aproximacion-a-su-utilizacion.pdf [Fecha de consulta: 22/03/2020].

Gijón, C.; Garín-Muñoz, T.; Pérez-Amaral, T.; López-Zorzano, R. (2013). Satisfaction of individual mobile phone users in Spain.Telecommunications Policy,37(10), 940-
954. https://doi.org/10.1016/j.telpol.2013.09.004 [Fecha de consulta: 09/02/2020].

Klimova, B. (2019). Impact of mobile learning on students' achievement results. Education Sciences, 9(2), 90. https://doi.org/10.3390/educsci9020090 [Fecha de consulta: 09/02/2020].

Krull, G.; Duart, J. M. (2018). Research Trends in Mobile Learning in Higher Education: A Systematic Review of Articles (2011-2015). International Review of Research in Open and Distributed Learning, 18(7), 1-23. https://doi.org/10.19173/irrodl.v18i7.2893 [Fecha de consulta: 09/02/2020].

López-Meneses, E., Bravo, C. B., Olivencia, J. J. L., \& Padilla, A. H. M. (2018). Validación del instrumento didáctico de valoración de observatorios digitales sobre MOOC: CUVOMOOC $\&$ mediante el Método Delphi. Campus Virtuales, 7(1), 95-110. http://dx.doi.org/10.17013/risti.32.1-16 [Fecha de consulta: 21/03/2020].

Mateus, J. C.; Aran-Ramspott, S.; Masanet, M. J. (2017). Revisión de la literatura sobre dispositivos móviles en la universidad española. RIED. Revista Iberoamericana de Educación a Distancia,20(2). https://doi.org/10.5944/ ried.20.2.17710 [Fecha de consulta: 09/02/2020].

Padilla, D. B.; Vázquez-Cano, E. ; Cevallos, M. B. M.; Meneses, E. L. (2019). Uso de apps de realidad aumentada en las aulas universitarias. Campus Virtuales, 8(1), 37-48. http://uajournals.com/ojs/index.php/campusvirtuales/ article/view/379 [Fecha de consulta: 09/02/2020].

Pechenkina, E. (2017). Developing a typology of mobile apps in higher education: A national case-study. Australasian Journal of Educational Technology, 33(4). [Fecha de consulta: 09/02/2020]. https://doi.org/10.14742/ ajet. 3228

Porcar, A. M.; Crespo, J. L. G.; Nuez, M. J. A. (2010). El mercado de las telecomunicaciones en España: un estudio sobre la evolución de la telefonía móvil. Boletín de Estudios Económicos,65(200), 361. http:// www.deusto-publicaciones.es/deusto/index.php/es/ component/content/article/61-revistas/148-revistasud04cast.html [Fecha de consulta: 09/02/2020].

Reguant Álvarez, M., \& Torrado Fonseca, M. (2016). El método Delphi. REIRE: Revista d'Innovació i Recerca en Educació, 2016, vol. 9, num. 2, p. 87-102. http://hdl.handle. net/2445/110707 [Fecha de consulta: 22/03/2020].

Sánchez, C. C. (2012). Estado del arte de la comunicación móvil en España. Aportaciones realizadas y retos de futuro. Vivat Academia,(117E), 1049-1058. https:// doi.org/10.15178/va.2011.117E.1049-1058 [Fecha de consulta: 09/02/2020].

Sung, Y. T.; Lee, H. Y.; Yang, J. M.; Chang, K. E. (2019). The quality of experimental designs in mobile learning research: A systemic review and self-improvement tool. Educational Research Review, 29,1-15. https:// doi.org/10.1016/j.edurev.2019.05.001 [Fecha de consulta: 09/02/2020].

Vassilakaki, E. (2014). Mobile information services in libraries: a review of current trends in delivering information. Interlending \& Document Supply, 42(4),176-186. https://doi.org/10.1108/ILDS-08-2014-0037 [Fecha de consulta: 09/02/2020].

Wai, I. S. H.; Ng, S. S. Y.; Chiu, D. K.; Ho, K. K.; Lo, P. (2018). Exploring undergraduate students' usage 
pattern of mobile apps for education. Journal of $\mathrm{Li}$ brarianship and Information Science, 50(1), 34-47. https://doi.org/10.1177/0961000616662699 [Fecha de consulta: 09/02/2020].
Witt, C.; Gloerfeld, C. (2018). Mobile Learning and Higher Education. En: The Digital Turn in Higher Education, pp. 61-79. Springer VS, Wiesbaden. https://doi. org/10.1007/978-3-658-19925-8_6 


\section{ANEXO I. UNIVERSIDADES ESPAÑOLAS}

\begin{tabular}{|c|c|c|c|}
\hline UNIVERSIDAD & ACRÓNIMO & ESTATUS & WEB \\
\hline IE Universidad & IE & Privada & www.ie.edu/universidad \\
\hline MondragónUnibertsitatea & UMON & Privada & www.mondragon.edu \\
\hline Universidad a Distancia de Madrid & UDIMA & Privada & www.udima.es \\
\hline Universidad Alfonso X El Sabio & UAX & Privada & www.uax.es \\
\hline Universidad Antonio de Nebrija & UANE & Privada & www.nebrija.com \\
\hline Universidad Autónoma de Barcelona & UAB & Pública & www.uab.es \\
\hline Universidad Autónoma de Madrid & UAM & Pública & www.uam.es \\
\hline Universidad Camilo José Cela & UCJC & Privada & www.ucjc.edu \\
\hline Universidad Cardenal Herrera-CEU & $\mathrm{UCH}$ & Privada & www.uchceu.es/ \\
\hline Universidad Carlos III de Madrid & UCAR & Pública & www.uc3m.es \\
\hline Universidad Católica de Valencia San Vicente Mártir & UCV & Privada & www.ucv.es \\
\hline Universidad Católica San Antonio & UCAM & Privada & www.ucam.edu \\
\hline Universidad Católica Santa Teresa de Jesús de Ávila & UCAV & Privada & www.ucavila.es \\
\hline Universidad Complutense de Madrid & UCM & Pública & www.ucm.es \\
\hline Universidad de A Coruña & UDC & Pública & www.udc.es \\
\hline Universidad de Alcalá & UAH & Pública & www.uah.es \\
\hline Universidad de Alicante & UA & Pública & www.ua.es/ \\
\hline Universidad de Almería & UAL & Pública & www.ual.es \\
\hline Universidad de Barcelona & UBA & Pública & www.ub.es \\
\hline Universidad de Burgos & UBU & Pública & www.ubu.es \\
\hline Universidad de Cádiz & UCA & Pública & www.uca.es \\
\hline Universidad de Cantabria & UCN & Pública & www.unican.es \\
\hline Universidad de Castilla-La Mancha & UCLM & Pública & www.uclm.es \\
\hline Universidad de Córdoba & UCO & Pública & www.uco.es \\
\hline Universidad de Deusto & UDE & Privada & www.deusto.es \\
\hline Universidad de Extremadura & UEX & Pública & www.unex.es \\
\hline Universidad de Girona & UDG & Pública & www.udg.es \\
\hline Universidad de Granada & UGR & Pública & www.ugr.es \\
\hline Universidad de Huelva & UHU & Pública & www.uhu.es \\
\hline Universidad de Jaén & UJA & Pública & www.ujaen.es \\
\hline Universidad de La Laguna & ULL & Pública & www.ull.es \\
\hline Universidad de La Rioja & UR & Pública & www.unirioja.es \\
\hline Universidad de Las Palmas de Gran Canaria & UPGC & Pública & www.ulpgc.es \\
\hline Universidad de León & ULE & Pública & www.unileon.es \\
\hline Universidad de Lleida & UDL & Pública & www.udl.es \\
\hline Universidad de Malaga & UMA & Pública & www.uma.es \\
\hline Universidad de Murcia & UMU & Pública & www.um.es \\
\hline Universidad de Navarra & UN & Privada & www.unav.es \\
\hline Universidad de Oviedo & UOV & Pública & www.uniovi.es \\
\hline Universidad de Salamanca & USAL & Pública & www.usal.es \\
\hline Universidad de Santiago de Compostela & USC & Pública & www.usc.es \\
\hline Universidad de Sevilla & USE & Pública & www.us.es \\
\hline
\end{tabular}




\begin{tabular}{|c|c|c|c|}
\hline UNIVERSIDAD & ACRÓNIMO & ESTATUS & WEB \\
\hline Universidad de Valladolid & UVA & Pública & www.uva.es \\
\hline Universidad de Vic-Universidad Central de Catalunya & UVIC & Privada & www.uvic.cat \\
\hline Universidad de Vigo & UVI & Pública & www.uvigo.gal \\
\hline Universidad de Zaragoza & UZA & Pública & www.unizar.es \\
\hline Universidad del Atlántico Medio & ATLANTICOMEDIO & Privada & www.universidatlanticomedio.es \\
\hline Universidad del País Vasco/EuskalHerrikoUnibertsitatea & UPV / EHU & Pública & www.ehu.eus \\
\hline Universidad Europea de Canarias & UEC & Privada & canarias.universidadeuropea.es \\
\hline Universidad Europea de Madrid & UEM & Privada & madrid.universidadeuropea.es \\
\hline Universidad Europea de Valencia & UEV & Privada & valencia.universidadeuropea.es \\
\hline Universidad Europea del Atlántico & UNEATLANTICO & Privada & www.uneatlantico.es \\
\hline Universidad Europea Miguel de Cervantes & UEMC & Privada & www.uemc.es \\
\hline Universidad Fernando Pessoa-Canarias & UFPC & Privada & www.ufpcanarias.es \\
\hline Universidad Francisco de Vitoria & UFV & Privada & www.ufv.es \\
\hline Universidad Internacional de Andalucía & UNIA & Pública & www.unia.es \\
\hline Universidad Internacional de La Rioja & UNIR & Privada & www.unir.net \\
\hline Universidad Internacional Isabel I de Castilla & UI1 & Privada & www.ui1.es \\
\hline Universidad Internacional Menéndez Pelayo & UIMP & Pública & www.uimp.es \\
\hline Universidad Jaume I de Castellón & UJI & Pública & www.uji.es \\
\hline Universidad Loyola Andalucía & ULA & Privada & www.uloyola.es \\
\hline Universidad Miguel Hernández de Elche & UMH & Pública & www.umh.es \\
\hline Universidad Nacional de Educación a Distancia & UNED & Pública & www.uned.es \\
\hline Universidad Pablo de Olavide & UPO & Pública & www.upo.es \\
\hline Universidad Politécnica de Cartagena & UPCT & Pública & www.upct.es \\
\hline Universidad Politécnica de Catalunya & UPC & Pública & www.upc.edu \\
\hline Universidad Politécnica de Madrid & UPM & Pública & www.upm.es \\
\hline Universidad PompeuFabra & UPF & Pública & www.upf.edu \\
\hline Universidad PontificiaComillas & COMILLAS & Privada & www.upcomillas.edu \\
\hline Universidad Pontificia de Salamanca & UPSA & Privada & www.upsa.es \\
\hline Universidad Pública de Navarra & UPNA & Pública & www.unavarra.es \\
\hline Universidad Ramón Llull & URLL & Privada & www.url.edu \\
\hline Universidad Rey Juan Carlos & URJC & Pública & www.urjc.es \\
\hline Universidad Rovira i Virgili & URV & Pública & www.urv.cat \\
\hline Universidad San Jorge & US] & Privada & www.usj.es \\
\hline Universidad San Pablo-CEU & UCEU & Privada & www.uspceu.com \\
\hline Universidad Tecnología y Empresa & UTE & Privada & www.usic.edu \\
\hline UniversitatAbatOliba CEU & UAO & Privada & www.uaoceu.com \\
\hline Universitat de les IllesBalears & UIB & Pública & www.uib.es \\
\hline Universitat de València (Estudi General) & UV & Pública & www.uv.es \\
\hline UniversitatInternacional de Catalunya & UIC & Pública & www.uic.es \\
\hline UniversitatInternacionalValenciana & VIU & Privada & www.universidadviu.com \\
\hline UniversitatOberta de Catalunya & UOC & Privada & www.uoc.edu \\
\hline UniversitatPolitècnica de València & UPV & Pública & www.upv.es \\
\hline
\end{tabular}

Registro de Universidades, Centros y Títulos. Ministerio de Ciencia, Innovación y Universidades.

https://www.educacion.gob.es/ruct/consultaestudios?actual=estudios 


\section{ANEXO II. ÁREAS FUNCIONALES Y PUNTUACIONES}

\begin{tabular}{|c|c|c|c|c|c|c|c|c|c|c|}
\hline \multirow{2}{*}{ UNIVERSIDAD } & \multirow{2}{*}{$\begin{array}{l}\text { No } \\
\text { de } \\
\text { apps }\end{array}$} & \multicolumn{8}{|c|}{ Areas funcionales y puntuaciones } & \multirow{2}{*}{$\begin{array}{l}\text { Puntuación } \\
\text { total }\end{array}$} \\
\hline & & Información & Gestión & Servicios & Biblioteca & e-Learning & Investigación & Movilidad & Promoción & \\
\hline UGR & 16 & 10 & 8 & 9 & & 5 & 5 & 7 & 12 & 56 \\
\hline UJA & 5 & 10 & 4 & 7 & & 9 & & 6 & 9 & 45 \\
\hline USAL & 2 & 10 & 2 & & 9 & 5 & & 7 & 9 & 42 \\
\hline UCM & 7 & 10 & 4 & 2 & & 9 & & 7 & 9 & 41 \\
\hline UCLM & 5 & 10 & 4 & 4 & & 9 & & 6 & 6 & 39 \\
\hline UPC & 5 & 10 & 2 & 5 & 5 & & 10 & & 6 & 38 \\
\hline UPM & 4 & 9 & 6 & 2 & 4 & & 10 & & 6 & 37 \\
\hline UPF & 4 & 9 & 5 & 2 & & 5 & & 7 & 9 & 37 \\
\hline UMH & 15 & 5 & 4 & 2 & & 10 & 5 & 10 & & 36 \\
\hline UMA & 6 & 10 & 7 & 4 & & 5 & & & 9 & 35 \\
\hline UEMC & 2 & 10 & 2 & & & 5 & & 7 & 9 & 33 \\
\hline COMILLAS & 2 & 10 & 2 & & & 5 & & 7 & 9 & 33 \\
\hline URJC & 1 & 10 & 2 & & & 5 & & 7 & 9 & 33 \\
\hline UCN & 4 & 10 & 2 & 4 & 4 & & & 6 & 6 & 32 \\
\hline UPCT & 2 & 9 & 4 & 4 & & 9 & & & 6 & 32 \\
\hline UA & 4 & 10 & 4 & & & 5 & & 6 & 6 & 31 \\
\hline UR & 2 & 10 & 4 & & 4 & & & 7 & 6 & 31 \\
\hline UDL & 1 & 10 & 2 & & & 5 & & 7 & 6 & 30 \\
\hline UVI & 1 & 10 & 2 & & & 5 & & 7 & 6 & 30 \\
\hline UCEU & 1 & 10 & 2 & & & 5 & & 7 & 6 & 30 \\
\hline UCAM & 1 & 10 & 2 & & & 5 & & 6 & 6 & 29 \\
\hline UHU & 1 & 10 & 2 & & & 5 & & 6 & 6 & 29 \\
\hline UPGC & 7 & 5 & 6 & & & 5 & & & 12 & 28 \\
\hline UMU & 8 & 10 & 7 & & & 5 & & & 6 & 28 \\
\hline UVA & 4 & & 8 & 2 & & 5 & & 7 & 6 & 28 \\
\hline UPSA & 1 & 10 & 2 & 3 & & & & 7 & 6 & 28 \\
\hline UCAR & 6 & 10 & & 6 & 4 & 4 & & & 3 & 27 \\
\hline ULE & 1 & 10 & 6 & & & 5 & & & 6 & 27 \\
\hline UBU & 1 & 10 & 2 & & & & & 7 & 6 & 25 \\
\hline UEM & 1 & 9 & 2 & 2 & & 5 & & 4 & 3 & 25 \\
\hline UV & 4 & 10 & 2 & & 8 & & & & 3 & 23 \\
\hline UOC & 8 & 6 & 2 & & & 9 & & & 6 & 23 \\
\hline UEX & 1 & 8 & 4 & 2 & & 5 & & & 3 & 22 \\
\hline UDG & 2 & 5 & 4 & 4 & & 9 & & & & 22 \\
\hline UPV / EHU & 5 & 9 & 2 & 8 & & & & & 3 & 22 \\
\hline UBA & 5 & 2 & 2 & 2 & & 9 & & & 6 & 21 \\
\hline USE & 1 & 6 & & 7 & & & 5 & & 3 & 21 \\
\hline UJI & 2 & 6 & 4 & & & 5 & & 3 & 3 & 21 \\
\hline UOV & 1 & 10 & 5 & & & 5 & & & & 20 \\
\hline
\end{tabular}




\begin{tabular}{|c|c|c|c|c|c|c|c|c|c|c|}
\hline \multirow{2}{*}{ UNIVERSIDAD } & \multirow{2}{*}{$\begin{array}{c}\text { No } \\
\text { de } \\
\text { apps }\end{array}$} & \multicolumn{8}{|c|}{ Areas funcionales y puntuaciones } & \multirow{2}{*}{$\begin{array}{l}\text { Puntuación } \\
\text { total }\end{array}$} \\
\hline & & Información & Gestión & Servicios & Biblioteca & e-Learning & Investigación & Movilidad & Promoción & \\
\hline UIB & 1 & 9 & 2 & 4 & & 5 & & & & 20 \\
\hline USC & 1 & 4 & 5 & 2 & & & 5 & & 3 & 19 \\
\hline URV & 2 & 9 & & 5 & 2 & & & & 3 & 19 \\
\hline UAX & 1 & 6 & & 3 & & & & 6 & 3 & 18 \\
\hline UDC & 1 & 6 & & 6 & & & & & 6 & 18 \\
\hline UCA & 12 & 6 & 4 & 2 & 3 & & & & 3 & 18 \\
\hline UAB & 3 & 8 & 4 & 2 & & & & & 3 & 17 \\
\hline UPV & 4 & 9 & 1 & 4 & & & & & 3 & 17 \\
\hline UAL & 1 & 5 & & 2 & & & & 9 & & 16 \\
\hline UCO & 5 & 5 & 4 & 2 & & 4 & & & & 15 \\
\hline UPNA & 2 & 8 & 7 & & & & & & & 15 \\
\hline URLL & 2 & 5 & 4 & 3 & & & & & 3 & 15 \\
\hline UCV & 1 & 4 & 2 & 4 & & 4 & & & & 14 \\
\hline UNIR & 2 & & 4 & 2 & & 5 & & & 3 & 14 \\
\hline IE & 2 & & & & 12 & & & & & 12 \\
\hline UMON & 1 & 5 & & 7 & & & & & & 12 \\
\hline UCAV & 1 & & & & 12 & & & & & 12 \\
\hline UN & 3 & 5 & 5 & 2 & & & & & & 12 \\
\hline UAM & 2 & 6 & 5 & & & & & & & 11 \\
\hline UVIC & 1 & 5 & 3 & & & & & & 3 & 11 \\
\hline UNED & 4 & 5 & & 3 & & & & & 3 & 11 \\
\hline UZA & 4 & & 2 & 2 & & & & & 6 & 10 \\
\hline ULL & 2 & & & 4 & & 2 & & & 3 & 9 \\
\hline UPO & 1 & 1 & 5 & & & & & & 3 & 9 \\
\hline UIMP & 1 & & 2 & & & & & & 6 & 8 \\
\hline UCJC & 2 & & & 2 & & & & & 3 & 5 \\
\hline UANE & 1 & & & & 4 & & & & & 4 \\
\hline Total & 212 & 449 & 193 & 142 & 71 & 202 & 40 & 166 & 288 & \\
\hline \multicolumn{2}{|l|}{ Peso ideal } & 10 & 10 & 20 & 12 & 13 & 10 & 10 & 15 & \\
\hline \multicolumn{2}{|c|}{ Numero de apps } & 57 & 53 & 39 & 12 & 35 & 6 & 25 & 52 & \\
\hline \multicolumn{2}{|l|}{ Peso minimo } & 1 & 1 & 2 & 2 & 2 & 5 & 3 & 3 & \\
\hline \multicolumn{2}{|l|}{ Peso medio } & 7.88 & 3.64 & 3.64 & 5.92 & 5.77 & 6.67 & 6.64 & 5.54 & \\
\hline \multicolumn{2}{|l|}{$\begin{array}{l}\text { Peso } \\
\text { maximo }\end{array}$} & 10 & 8 & 9 & 12 & 10 & 10 & 10 & 12 & \\
\hline
\end{tabular}


ANEXO III. MARCADORES IDEAL, MÁXIMO, MÍNIMO Y PROMEDIO SEGÚN ÁREA FUNCTIONAL

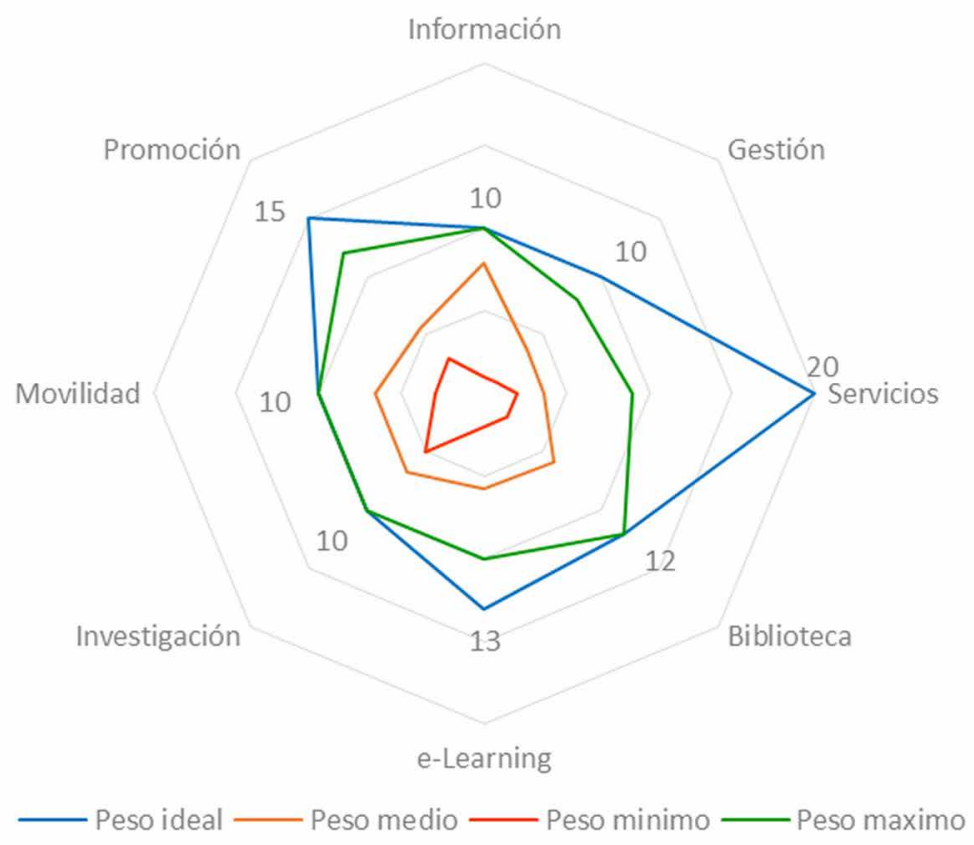

